\title{
Investigation of the Equivalent Test Condition for the Seismic Safety Assessment of a Spent Fuel Pool with regard to Sloshing Behavior
}

\author{
Won Man Park, ${ }^{1}$ Sung Man Son, ${ }^{1}$ Dae Kyung Choi, ${ }^{1}$ Se Hong Oh, ${ }^{1}$ Kang Hee Lee, \\ Heung Seok Kang, ${ }^{2}$ and Choengryul Choi $\mathbb{i}^{1}$ \\ ${ }^{1}$ Elsoltec Inc., Yongin, Republic of Korea \\ ${ }^{2}$ Korea Atomic Energy Research Institution, Daejeon, Republic of Korea \\ Correspondence should be addressed to Choengryul Choi; crchoi@elsoltec.com
}

Received 1 July 2019; Revised 8 October 2019; Accepted 1 November 2019; Published 1 December 2019

Academic Editor: Leon Cizelj

Copyright (C) 2019 Won Man Park et al. This is an open access article distributed under the Creative Commons Attribution License, which permits unrestricted use, distribution, and reproduction in any medium, provided the original work is properly cited.

\begin{abstract}
Spent fuel pools are used as temporary storage for spent fuel assemblies in nuclear power plants and are filled with coolant which removes the decaying heat from spent fuel assemblies. Sloshing of the coolant can occur if an earthquake occurs in the area. It may produce additional forces on the pool or inner structure and cause overflow of the coolant. It is therefore critical to investigate the phenomenon of sloshing in a seismic assessment of the spent fuel pool. The size of an actual spent fuel pool is excessive for carrying out an experimental study; thus, a scale model is necessary for experimentation. In this study, a scaling law was defined for test conditions using a scale model to understand sloshing behavior, and the results were validated via computational fluid dynamic analysis. Because sloshing is resonant in a fluid and the first mode natural frequency of a fluid is dominant in sloshing behavior, the test condition could be obtained based on the natural frequency of the fluid. In the model, which is scaled with a factor of " $S_{f}$," the scale factors " $S_{f}$, " " $S_{f}^{0}$ " " $S_{f}^{-0.5}$," and " $S_{f}^{0.5}$ " were used for displacement, acceleration, excitation frequency, and excitation time, respectively. Approximately $5 \%$ difference in maximum sloshing height between two models was predicted in the only case that $1 / 8$ and $1 / 4$ models $(1 / 8$ and $1 / 4$ scaled down from an actual spent fuel pool) were excited with $10 \mathrm{~Hz}$ and $7.071 \mathrm{~Hz}$, respectively, but the same sloshing height and pressure were predicted in other cases. The results of this study support the idea that the Froude scaling law can be used when using a scale model for a seismic assessment of spent fuel pools to investigate sloshing behavior.
\end{abstract}

\section{Introduction}

Spent fuel pools are facilities in nuclear power plants that act as temporary onsite storage for spent fuel assemblies which have been used in the nuclear reactor. Spent fuel assemblies produce heat from radioactive decay which could result in a critical accident and therefore need to be removed by the coolant within a spent fuel pool. Otherwise, it has been reported that the generation of hydrogen gas caused by the melting of the zircaloy cladding could lead to an explosion in the spent fuel pool [1-3]. Therefore, one of the major concerns about the spent fuel pool is its ability for preventing the loss of coolant and thus maintaining cooling of the radioactive fuel.
When an external force such as a seismic wave shakes a tank or pool which contains fluid, it could lead to sloshing behavior of the fluid. In the case of the spent fuel pool, the sloshing behavior of the coolant causes additional force on the wall of the pool and the racks [4]. This sloshing can also cause overflow of the coolant from the pool, resulting in a reduction in the amount of coolant. For instance, the USNR Commission has reported that decreases in the coolant level by $2.5 \mathrm{ft}(1.5 \mathrm{~m})$ was estimated at Units 1 and 3 of the Fukushima Daiichi plant after the Tohoku earthquake (March 11, 2011, Japan) [1]. This overflow, in the authors' opinion, could be crucial with regard to cooling of the spent fuel assemblies; therefore, it is critical to investigate the sloshing behavior of coolant in an evaluation 
of the effects seismic occurrences may have on a spent fuel pool.

Sloshing behavior can be investigated using analytical, numerical, and experimental methods [5-8]. The results of experimental studies are used for investigating the sloshing behaviors as well as for validating the results of numerical and analytical studies. However, spent fuel pools are too large to be investigated using a model of actual scale. For example, the size of the spent fuel pool at a particular nuclear power plant in Korea is known to be $10.4 \mathrm{~m} \times 8.6 \mathrm{~m} \times 12.8 \mathrm{~m}$. The use of a scaled-down model and appropriate test conditions for the model are therefore necessary for investigating sloshing behavior in the spent fuel pool.

Sloshing has been investigated in the seismic assessment of nuclear power plants as well as in marine and ocean engineering because of its importance in fluid dynamics. Faltinsen introduced a mathematical model for estimating sloshing motion in a rectangular tank [5]. Various numerical models have been developed by Frandsen, Wu et al., Chen et al., and Okamoto and Kawahara and used for investigating sloshing motion in various excitation conditions [6, 9-11]. Goudarzi and Sabbagh-Yazdi investigated the sloshing behavior of a spent fuel pool for a seismic safety assessment via experiments using scale models and computer simulation [12]. Zhao et al. Mitra and Sinhamahapatra developed a pressure-based finite element technique and analyzed the sloshing dynamics of a partially filled rigid container with bottom-mounted submerged components [13]. Kim et al. investigated sloshing-induced pressure in tanks of different scales by using an experimental method to investigate the pressure applied on the tanks of liquid natural gas (LNG) carriers under conditions of the movement of the ship [14]. Merino et al. analyzed the motion of a submerged rack, on which sloshing behavior can affect, in a spent fuel pool as a response to seismic movement using scale models via experimental methods and computer simulation in the assessment of a spent fuel pool [15].

In case that an actual scale model is too big or too small, the use of a scaled-down or -up model instead is very common in the mechanical engineering field $[12,14-16]$. An actual "spent fuel pool," which is the interest of this study, is not only too big but also too heavy to be used as experimental equipment. Therefore, for predicting and investigating the sloshing behavior of the coolant in a spent fuel pool, it is necessary to develop a scaled-down model and experimental conditions for it. Here, the methods to develop a scaled model and test conditions are important factors determining the accuracy and validity of the experiments. In this study, the test conditions were calculated to investigate sloshing behavior in a scaleddown model which has the same shape but is different in size to an actual pool. The conditions were validated via computer simulation by using computational fluid dynamics. The developed testing condition was compared to the testing condition using dimensionless numbers. Moreover, the sloshing behavior of the coolant in a spent fuel pool was evaluated under beyond-design-earthquake (BDE) conditions.

\section{Materials and Methods}

2.1. Test Condition for the Sloshing Behavior of the Coolant in the Scale Model. It has previously been observed that the first natural frequency and mode are dominant in the sloshing motion of a fluid in a tank or pool $[17,18]$. The first natural frequency of a fluid can be calculated via the following [5]:

$$
\begin{aligned}
& \omega_{n}^{2}=g k_{n} \tanh \left(k_{n} h\right), \\
& k_{n}=\frac{2 n+1}{L} \pi, \quad n=0,1,2,3, \ldots,
\end{aligned}
$$

where $\omega_{n}, g, h, L$, and $k_{n}$ are the nth natural frequency (rad/ s) of the fluid, gravity, depth of fluid, width of a tank or pool, and wave number, respectively.

When $n$ is $0, \tanh \left(k_{n} h\right)$ is calculated as 0.999 and it becomes 1.000 when $n$ is greater than or same to 1 . Therefore, we can assume that the square root of the natural frequency is inversely proportional to $L$ and $L$ is directly proportional to the scale factor $S_{f}$ :

$$
\begin{aligned}
\omega_{n}^{2} & \approx g k_{n}, \\
\therefore \omega_{n}^{2} & \propto \frac{1}{L}, \\
S_{f} & \propto L .
\end{aligned}
$$

Therefore, the square root of the natural frequency is inversely proportional to the scale factor $S_{f}$ :

$$
\omega_{n} \propto \frac{1}{\sqrt{S_{f}}} .
$$

Because the sloshing behavior of the fluid resonates with external excitation, it is to be expected that the external excitation of the scaled-down model is

$$
\omega_{\text {scale }}=\frac{1}{\sqrt{S_{f}}} \omega,
$$

where $\omega$ and $\omega_{\text {scale }}$ are the excitation frequency ( $\left.\mathrm{rad} / \mathrm{s}\right)$ for the actual size and the scaled-down model, respectively.

Based on the linear theory introduced by Faltinsen, the sloshing height $(\xi)$ of a two-dimensional rectangular tank at location $x$ and time $t$ can be calculated as follows:

$$
\xi(x, t)=\xi_{1}(x, t)+\xi_{2}(x, t),
$$

where $\xi_{1}$ and $\xi_{2}$ are the impulsive and convective components of sloshing, calculated from the following equations $[9,12]$ :

$$
\begin{aligned}
& \xi_{1}(x, t)=\frac{A}{g}\left(x \omega^{2}+\sum_{n=0}^{\infty} C_{n} \cdot \omega \cdot \sin \left(k_{n} x\right)\right) \sin (\omega t), \\
& \xi_{2}(x, t)=\frac{A}{g} \sum_{n=0}^{\infty} \omega_{n}\left(C_{n}+\frac{H_{n}}{\omega^{2}}\right) \sin \left(k_{n} x\right) \sin \left(\omega_{n} t\right),
\end{aligned}
$$

where $A$ and $g$ are the amplitudes of excitation and gravity, respectively. Parameters in equations (7) and (8), $\omega_{n}, k_{n}, C_{n}$, 
and $H_{n}$, are defined according to equations (1), (2), (9), and (10):

$$
\begin{aligned}
& H_{n}=\omega^{3}\left(\frac{4}{L}\right)\left(\frac{(-1)^{n}}{k_{n}^{2}}\right), \\
& C_{n}=\frac{H_{n}}{\omega_{n}^{2}-\omega^{2}} .
\end{aligned}
$$

Because both $\xi_{1}$ and $\xi_{2}$ are directly proportional to amplitude $A$ and $A$ is directly proportional to the scale factor, $\xi$ is directly proportional to the scale factor $S_{f}$.

Thus, the excitation frequency $\left(\omega_{\text {scale }}\right)$, amplitude $\left(A_{\text {scale }}\right)$, and excitation time $\left(t_{\text {scale }}\right)$ of the scaled-down model are as follows:

$$
\begin{aligned}
\omega_{\text {scale }} & =\frac{1}{\sqrt{S_{f}}} \omega, \\
A_{\text {scale }} & =S_{f} \cdot A, \\
\text { (duration) } t_{\text {scale }} & =\sqrt{S_{f}} \cdot t .
\end{aligned}
$$

If it is assumed that if sine-wave external excitation is applied, the displacements of the actual scale model $(d(t))$ and the scaled-down model $\left(d(t)_{\text {scale }}\right)$ are calculated as follows:

$$
\begin{aligned}
d(t) & =A \cdot \sin (\omega \cdot t), \\
d(t)_{\text {scale }} & =A_{\text {scale }} \cdot \sin \left(\omega_{\text {scale }} \cdot t_{\text {scale }}\right) \\
& =A \cdot S_{f} \cdot \sin \left(\frac{1}{\sqrt{S_{f}}} \omega \cdot t\right) .
\end{aligned}
$$

If external excitation is applied using acceleration, the acceleration of the real scale model $(a(t))$ and the scaleddown model $\left(a(t)_{\text {scale }}\right)$ are calculated as follows:

$$
a(t)=-A \cdot \omega^{2} \cdot \sin (\omega \cdot t)
$$

$$
\begin{aligned}
a(t)_{\text {scale }} & =d_{\text {scale }}^{\prime \prime}=A \cdot S_{f} \cdot(-1) \cdot\left(\frac{\omega}{\sqrt{S f}}\right)^{2} \cdot \sin \left(\frac{1}{\sqrt{S_{f}}} \omega \cdot t\right) \\
& =-A \cdot \omega^{2} \cdot \sin \left(\frac{1}{\sqrt{S_{f}}} \omega \cdot t\right) .
\end{aligned}
$$

The amplitude of the excitation acceleration for the scaled-down model is therefore the same as that of the actual scale model, but the duration of the excitation time in the scaled-down model should be changed by considering the scale factor given in equation (13).

2.2. CFD (Computational Fluid Dynamics) Analysis of the Sloshing Behaviors. The length, width, and height of the spent fuel pool at the Korean nuclear power plant considered in this study are approximately $10.4 \mathrm{~m}, 8.6 \mathrm{~m}$, and $12.8 \mathrm{~m}$, respectively. The level of the coolant reaches approximately $12.2 \mathrm{~m}$ in the pool. Two-dimensional computer fluid dynamic models with scale factors of $1 / 4$ and $1 / 8$ were developed using the commercial CFD software Ansys/Fluent (Ansys Inc., Canonsburg, PA, US) (Figure 1). However, the height of the pool was enlarged as high as twice the water level to avoid overflow of the water, because we could not obtain the sloshing height in case that the sloshing motion exceeds the given analysis domain. Thus, the length and height of the pool and the water level of the $1 / 8$ and $1 / 4$ scale models were assumed to be $1.3 \mathrm{~m}, 3.0 \mathrm{~m}$, and $1.525 \mathrm{~m}$ (for the $1 / 8$ scale model) and $2.6 \mathrm{~m}, 6.0 \mathrm{~m}$, and $3.050 \mathrm{~m}$ (for the $1 /$ 4 scale model), respectively.

Each model consists of water (instead of the boric acid coolant in the scale model) and air. The temperature of the water and air was set at $25^{\circ} \mathrm{C}$. Densities of $997 \mathrm{~kg} / \mathrm{m}^{3}$ and $1.185 \mathrm{~kg} / \mathrm{m}^{3}$ and viscosities of $8.899 \times 10^{-4} \mathrm{~kg} /(\mathrm{m} \cdot \mathrm{s})$ and $1.831 \times 10^{-5} \mathrm{~kg} /(\mathrm{m} \cdot \mathrm{s})$ were used for water and air, respectively. The water was considered as an incompressible fluid. A surface tension of $0.07199 \mathrm{~N} / \mathrm{m}$ was applied for the water. A no-slip boundary condition was used at the interface between the wall of the pool and the fluid (water and air). An opening boundary condition for the superior plane of the air (the top plane of the domain) was used, and the opening pressure was set to be $0 \mathrm{~Pa}$. Although $\mathrm{Ha}$ et al. have reported that the shear stress transport model showed better results in predicting the sloshing motion compared to the standard $k-\varepsilon$ model [19], the standard $k-\varepsilon$ model is widely used even in the prediction of sloshing motion because it shows much faster convergence than others $[20,21]$. Thus, the standard $k$ - $\varepsilon$ turbulence model was used to minimize the computing time in this study. In total, 156,000 elements (260 elements along the horizontal direction and 600 elements along the vertical direction) were used for both models.

The volume of fluid (VOF) method was used to find the free surface motion of the liquid sloshing in the pool. Thus, the free surface is identified using a characteristic function $F(x, y, t)$, which represents the volume fraction of the fluid at each cell. $F$ is calculated as a number between 0 and 1 , where the cell is fully filled with air or water, respectively, and $0<F<1$ describes a cell that is partially filled with water, identifying the location of the free surface of the water.

For the 1/8 model, a rigid pool was horizontally excited for $5 \mathrm{~s}$ at frequencies of $1 \mathrm{~Hz}$ and $10 \mathrm{~Hz}$ with an amplitude of $5 \mathrm{~mm}$ used for the excitation. Sloshing behaviors were shown in limited range regardless of the increase in excitation frequency in the previously published numerical study, while the behaviors increased with resonating the excitation frequency when the excitation frequency was similar to the first natural frequency of the contained fluid [18]. The first natural frequency of the contained water of the $1 / 8$ model was about $0.77 \mathrm{~Hz}$. Thus, $1 \mathrm{~Hz}$ and $10 \mathrm{~Hz}$ were chosen for the excitation frequencies; one is around the first natural frequency of the fluid, and the other is much greater than it. The excitation frequency, amplitude, and excitation time were calculated using equations (11)-(13). The pool used for the 1/ 4 model was excited with frequencies of $0.707 \mathrm{~Hz}$ and $7.071 \mathrm{~Hz}$ for $7.07 \mathrm{~s}$ (Table 1) with an amplitude of $10 \mathrm{~mm}$. 


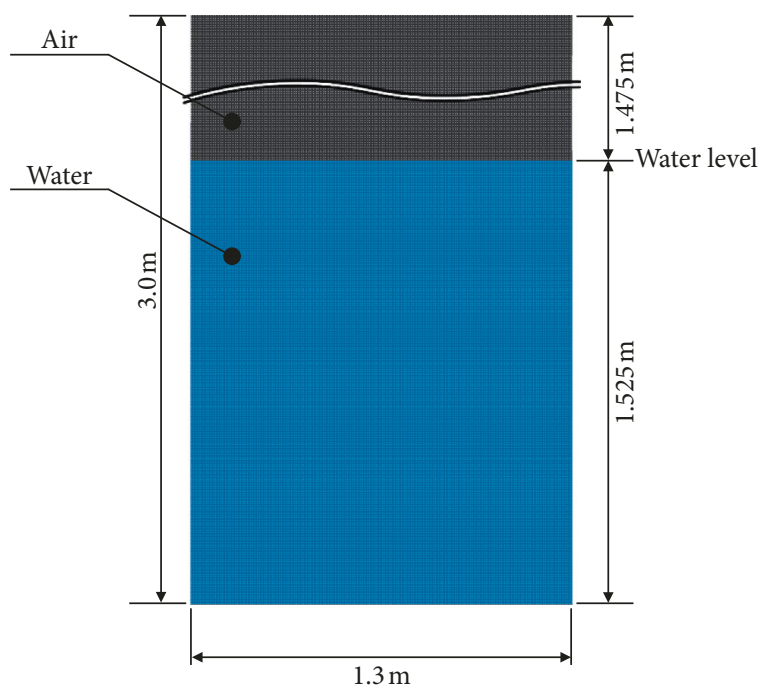

FIGURE 1: CFD model developed for the spent fuel pool with a scale factor of $1 / 8$.

TABLe 1: Conditions for CFD analysis in the $1 / 4$ - and $1 / 8$-scaled models.

\begin{tabular}{lccc}
\hline No. & Model scale & $\begin{array}{c}\text { Excitation (type: frequency, } \\
\text { amplitude) }\end{array}$ & $\begin{array}{c}\text { Duration of } \\
\text { analysis }\end{array}$ \\
\hline 1 & $1 / 8$ & Sine wave: $1 \mathrm{~Hz}, 5 \mathrm{~mm}$ & $5 \mathrm{~s}$ \\
2 & $1 / 4$ & Sine wave: $0.707 \mathrm{~Hz}, 10 \mathrm{~mm}$ & $7.07 \mathrm{~s}$ \\
3 & $1 / 8$ & Sine wave: $10 \mathrm{~Hz}, 5 \mathrm{~mm}$ & $5 \mathrm{~s}$ \\
4 & $1 / 4$ & Sine wave: $7.071 \mathrm{~Hz}, 10 \mathrm{~mm}$ & $7.07 \mathrm{~s}$ \\
5 & $1 / 8$ & BDE data & $5 \mathrm{~s}$ \\
6 & $1 / 4$ & BDE data & $7.07 \mathrm{~s}$ \\
\hline
\end{tabular}

The duration of analysis ( $5 \mathrm{~s}$ for $1 / 8$ model and $7.07 \mathrm{~s}$ for $1 / 4$ model) corresponds to $14.14 \mathrm{~s}$ for the actual model.

Both models were also tested under BDE conditions. Time history ground acceleration data were artificially generated for $20 \mathrm{~s}$ based on the R. G. 1.60 spectrum [22]. The maximum value of ground acceleration used for BDE was set at $0.3 \mathrm{~g}$ (Figure 2). Because the time history acceleration data were generated for the full-scale model, the data were scaled for both the 1/8 and $1 / 4$ scale models. The amplitude and excitation time for both models were calculated using equations (13) and (16), respectively. The same amplitude was used for both models as that used in the full-scale model, and the excitation time was scaled by $1 / 8^{0.5}$ and $1 / 4^{0.5}$. The $1 /$ 8 model was then excited for $5 \mathrm{~s}$ (of $7.071 \mathrm{~s}$ scaled from $20 \mathrm{~s}$ by $1 / 8^{0.5}$ ) and the $1 / 4$ model for $7.071 \mathrm{~s}$ (of $10 \mathrm{~s}$ scaled by $1 /$ $4^{0.5}$ ) (Table 1).

Prior to investigating equivalent test conditions for the scale models, three $1 / 8$ models and two $1 / 4$ models were developed with different mesh sizes for the mesh sensitivity study (Table 2). Sloshing heights were estimated in the developed $1 / 8$ and $1 / 4$ models. $1 / 8$ models were tested under excitation frequencies of $1 \mathrm{~Hz}$ and $10 \mathrm{~Hz}$ with an amplitude of $5 \mathrm{~mm}$ and $1 / 4$ models under an excitation frequency of $10 \mathrm{~Hz}$ with an amplitude of $10 \mathrm{~mm}$. All models were tested during $3 \mathrm{~s}$, and the predicted sloshing heights were compared with each other.

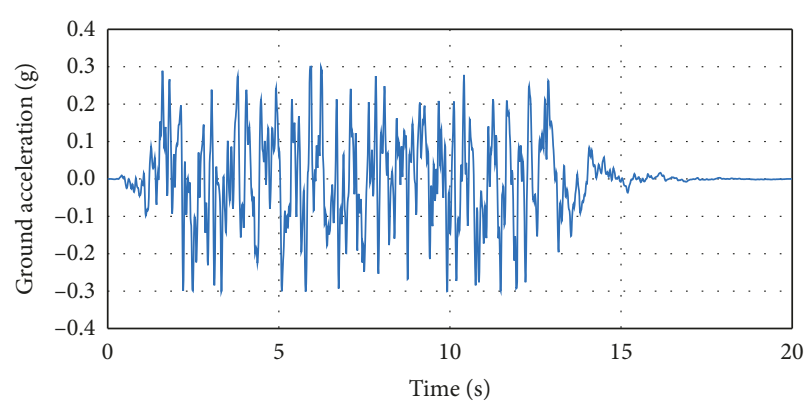

FIgURE 2: Artificially generated ground acceleration time history for design basis earthquake.

\section{Results}

When $1 / 8$ models were tested under an excitation frequency of $1 \mathrm{~Hz}$, the same changes in sloshing height were computed in three models (coarse, medium, and fine). During the whole analysis time, the medium model showed lower sloshing height than that of the fine model by $2 \mathrm{~mm}$, while and coarse model showed greater height by $4 \mathrm{~mm}$. When the models were tested with an excitation frequency of $10 \mathrm{~Hz}$, local differences were predicted. The maximum differences were $137 \mathrm{~mm}$ between the medium and fine models and $165 \mathrm{~mm}$ between the coarse and fine models. The medium and fine $1 / 4$ models also showed local differences as shown in $1 / 8$ models. The maximum difference between the two models was $216 \mathrm{~mm}$. In spite of the differences, all $1 / 8$ models and $1 / 4$ models showed the same trends of changes in sloshing height (Figure 3). Thus, medium $1 / 8$ and $1 / 4$ models were chosen.

The changes in sloshing height on the left wall of the $1 / 8$ model with an excitation frequency of $1 \mathrm{~Hz}$ (low frequency) and an amplitude of $5 \mathrm{~mm}$ was compared to the results of the $1 / 4$ model to which the corresponding excitation was applied (Figure 4). The maximum sloshing height in the $1 / 8$ model was $32.3 \mathrm{~mm}$ and $64.9 \mathrm{~mm}$ in the $1 / 4$ model. Sloshing heights were normalized using the initial water levels and compared with each other. The results from the $1 / 8$ and $1 / 4$ models were found to show good agreement.

Changes in the sloshing height on the left wall of the pool were also compared with the high excitation frequency of $10 \mathrm{~Hz}$ applied to the $1 / 8$ model and $7.071 \mathrm{~Hz}$ to the $1 / 4$ model (Figure 5). Approximately 5\% difference in maximum sloshing height was predicted for the two models. However, the overall sloshing motions of the two models were similar. Sloshing heights were also compared under conditions of BDE excitation (Figure 6). Both models showed very similar results, and the difference in the normalized maximum sloshing height was less than $1 \%$.

Changes in the pressure on the left wall of the pool were predicted at a height of $0.05 \mathrm{~m}$ and $1.4 \mathrm{~m}$ in the $1 / 8$ model and at a height of $0.1 \mathrm{~m}$ and $2.8 \mathrm{~m}$ in the $1 / 4$ model. Because the $1 / 4$ model is double the scale of the $1 / 8$ model, predicted pressures in the $1 / 8$ model were doubled for comparison to those in the 1/4 model under normalized time (Figure 7). In all testing conditions, both models showed the same changes in pressures at both heights. 
TABLE 2: Developed CFD models for mesh sensitivity study.

\begin{tabular}{lccc}
\hline & & Number of elements (width $\times$ height) & Element size $($ width $\times$ height $)$ \\
\hline \multirow{3}{*}{$1 / 8$ model } & Coarse & $130 \times 300$ & $10 \mathrm{~mm} \times 10 \mathrm{~mm}$ \\
& Medium & $260 \times 600$ & $5 \mathrm{~mm} \times 5 \mathrm{~mm}$ \\
\multirow{2}{*}{$1 / 4$ model } & Fine & $390 \times 900$ & $3.33 \mathrm{~mm} \times 3.33 \mathrm{~mm}$ \\
& Medium & $260 \times 600$ & $10 \mathrm{~mm} \times 10 \mathrm{~mm}$ \\
& Fine & $520 \times 1200$ & $5 \mathrm{~mm} \times 5 \mathrm{~mm}$ \\
\hline
\end{tabular}

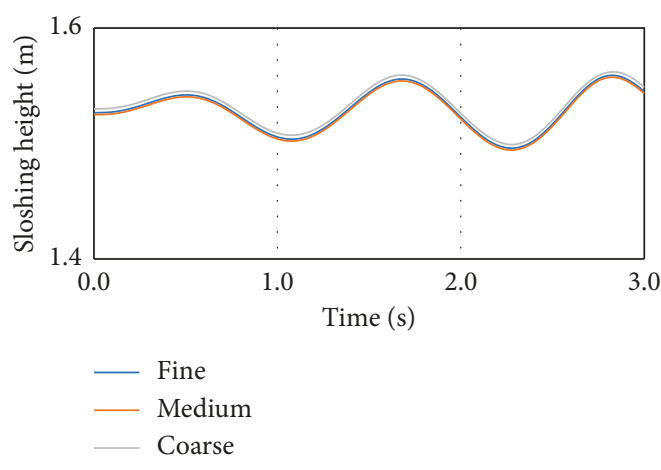

(a)

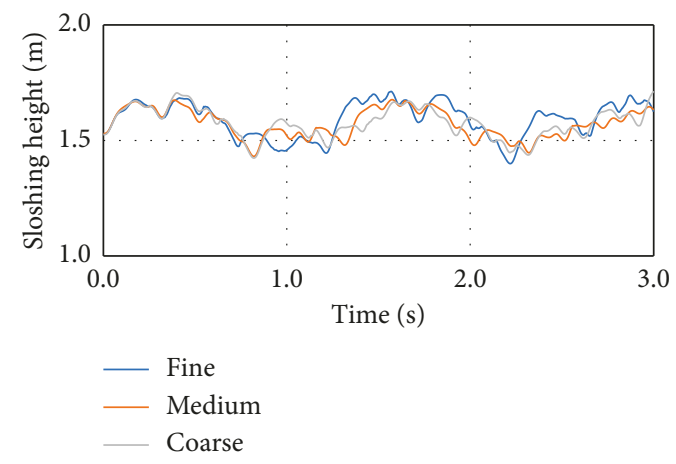

(b)

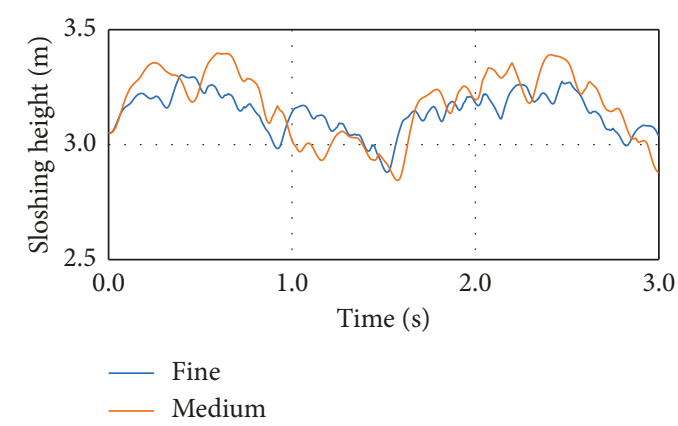

(c)

Figure 3: Comparison of sloshing for the sensitivity study. Sloshing heights in (a) $1 / 8$ models under $1 \mathrm{~Hz}$, (b) $1 / 8$ models under $10 \mathrm{~Hz}$, and (c) $1 / 4$ models under $10 \mathrm{~Hz}$.

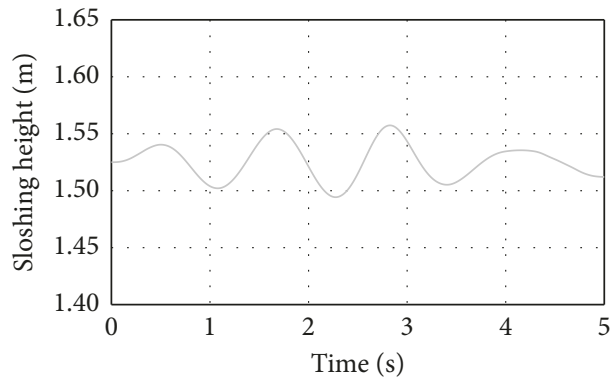

(a)

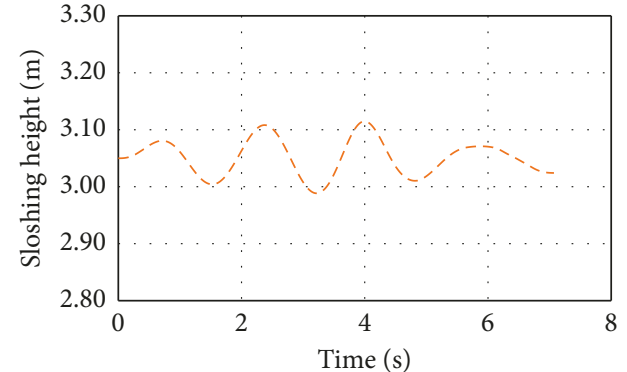

(b)

Figure 4: Continued. 


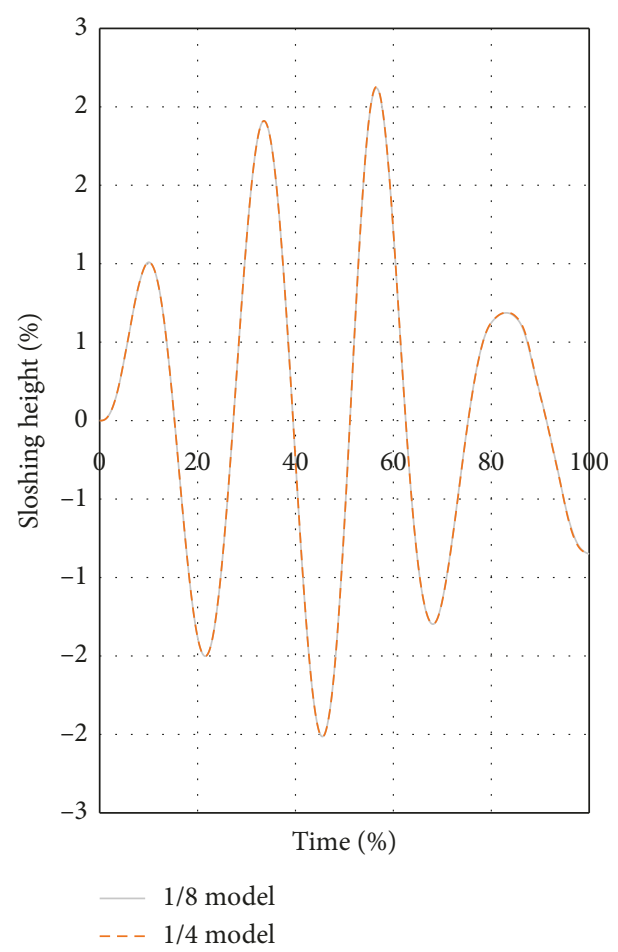

(c)

FIGURE 4: Sloshing heights in the (a) 1/8 model and (b) 1/4 model. (c) Comparison of sloshing heights under low-frequency excitation.

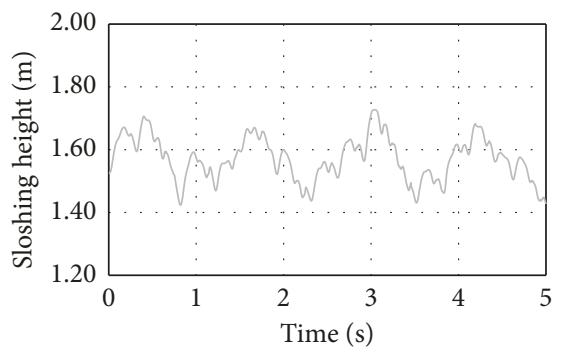

(a)

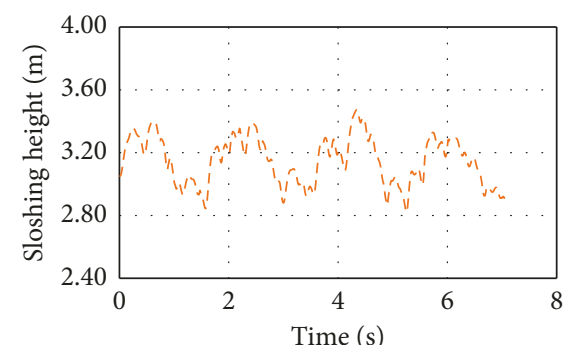

(b)

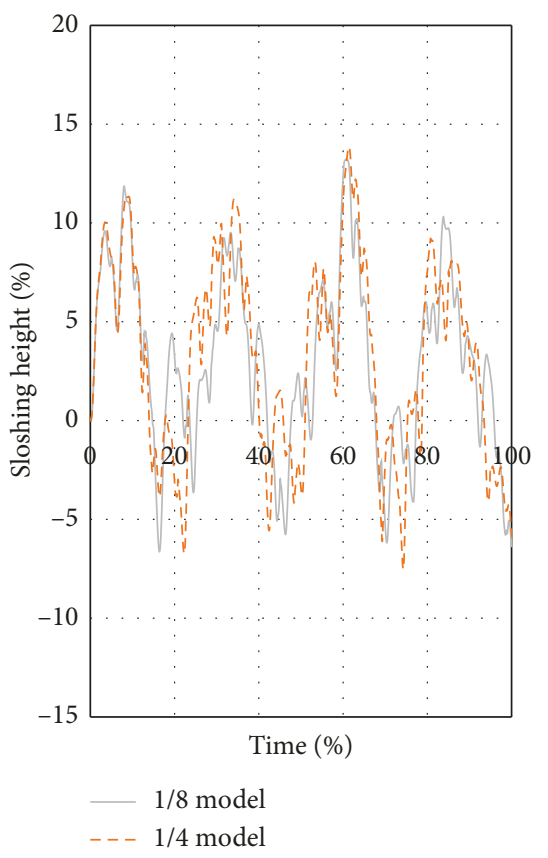

(c)

FIGURE 5: Sloshing heights in (a) 1/8 model and (b). 1/4 model. (c) Comparison of sloshing heights under high-frequency excitation. 


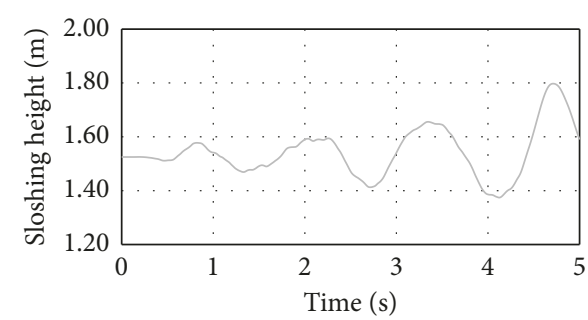

$1 / 8$ model

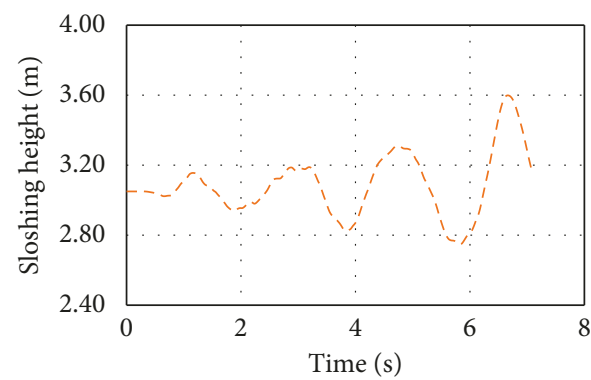

(b)

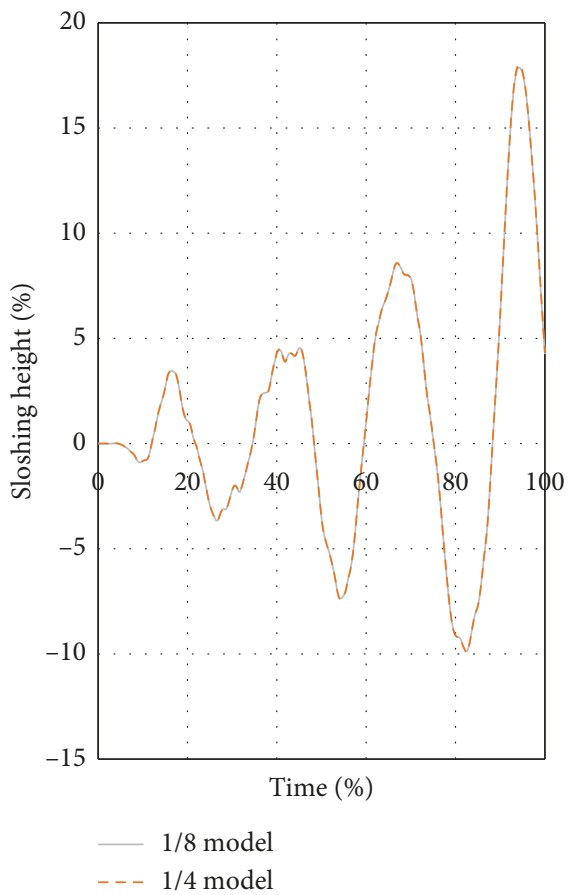

(c)

FIGURE 6: Sloshing heights in (a) 1/8 mode and (b) 1/4 model. (c) Comparison of sloshing heights under BDE.

\section{Discussion}

Several nondimensional numbers such as the Reynolds number, Froude number, Mach number, and Weber number have been used for developing scale models and obtaining suitable experimental conditions. The Froude number, which is defined as the ratio of flow inertia $\left(F_{i}\right)$ to gravity $\left(F_{g}\right)$ in continuum mechanics, has been used for studying fluid behavior using scale models $[14,23,24]$ :

$$
\frac{\text { inertia force }}{\text { gravity force }}=\frac{F_{i}}{F_{g}} \propto \frac{\rho U^{2} L^{2}}{\rho g L^{3}}=\frac{U^{2}}{g L},
$$

where, $\rho, U, L$, and $g$ are density, characteristic flow velocity, characteristic length, and acceleration due to gravity, respectively. The dynamic similarity requirement between real and scale models could be satisfied using equality in the Froude number $(F n)$, shown as follows:

$$
\begin{aligned}
\frac{U_{M}^{2}}{g L_{m}} & =\frac{U_{F}^{2}}{g L_{F}}, \\
\frac{U_{M}}{\sqrt{g L_{M}}} & =\frac{U_{F}}{\sqrt{g L_{F}}}=F n .
\end{aligned}
$$

The relevant scale factors for Froude similarity have been given in previously published research (Table 3 ) $[25,26]$. The calculated test conditions for the acceleration, velocity, length, and duration of sloshing behavior based on the natural frequencies of the fluid in the spent fuel pool were equal to the scale factors from the Froude similarity. When the Froude number is maintained in a scale model, the Weber number, which is the ratio of inertial force to surface tension force in equation (19), can be identical in models of different sizes, while the Reynolds number, which is the ratio of inertial force to viscous force in equation (20), cannot be identical: 
Height at $0.05 \mathrm{~m}$ of $1 / 8$ model (at $0.1 \mathrm{~m}$ of $1 / 4$ model)
Height at $1.4 \mathrm{~m}$ of $1 / 8$ model

(at $2.8 \mathrm{~m}$ of $1 / 4$ model)

Low-excitation frequency
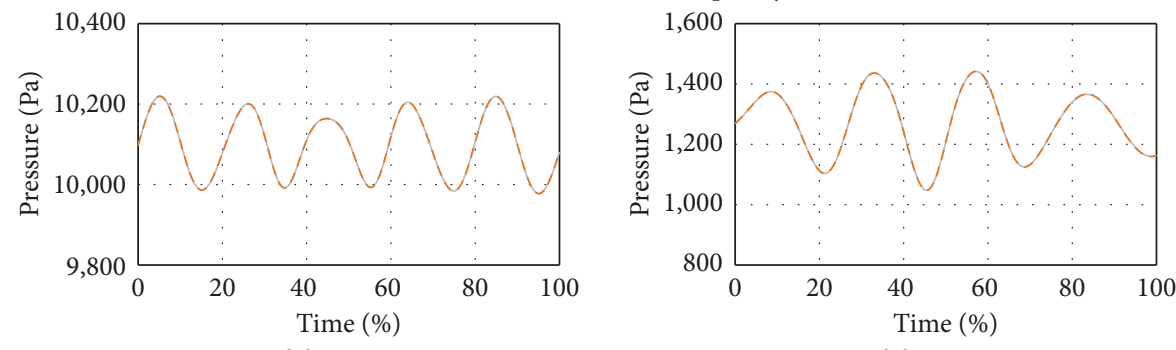

1/8 mode

- 1/8 model

- - $1 / 4$ model

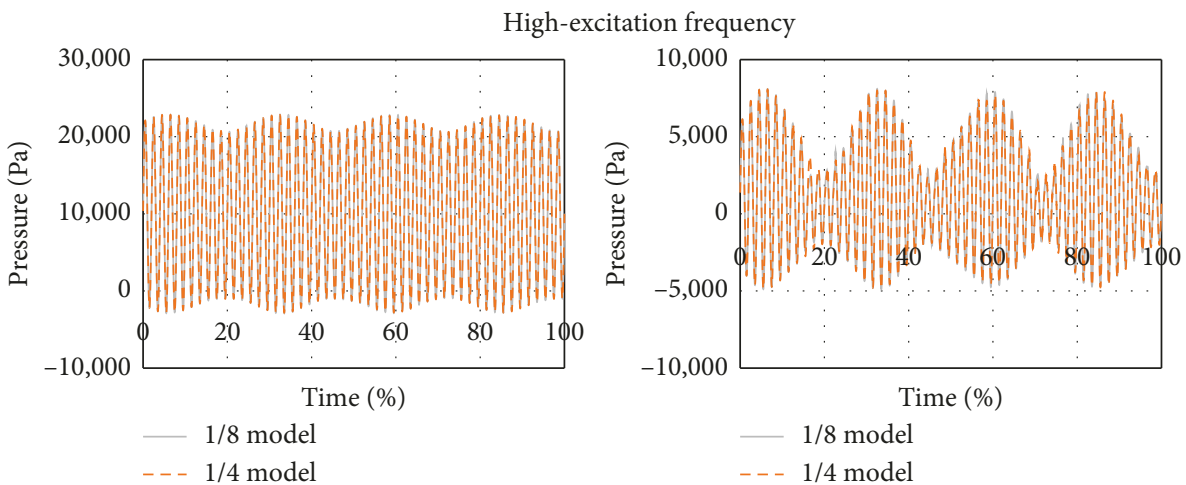

DBE conditions
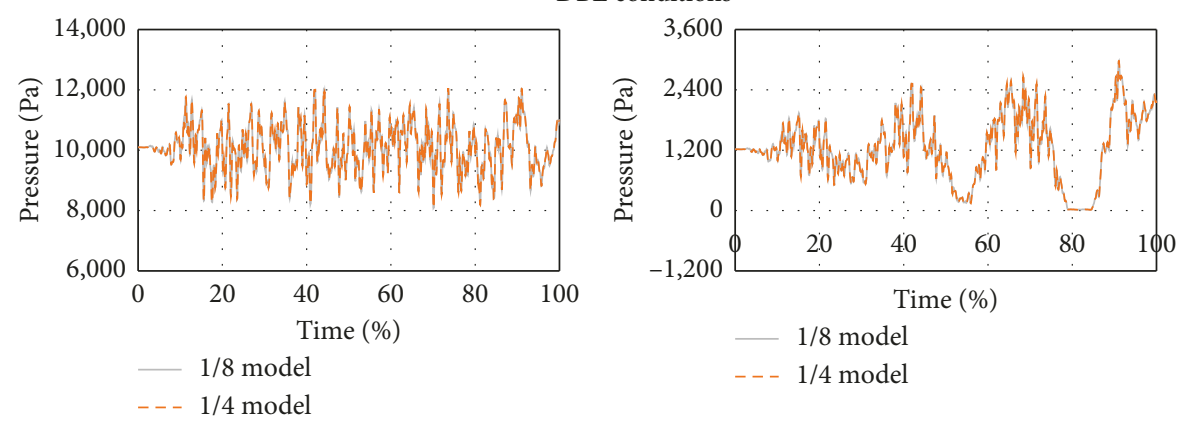

FIGURE 7: Comparison of the pressure at two different heights.

TABLE 3: Scale factors by Froude similarity.

\begin{tabular}{lc}
\hline Quantity & Scale factor \\
\hline Acceleration & $S_{f}^{0}$ \\
Area & $S_{f}^{2}$ \\
Mass/volume/force & $S_{f}^{3}$ \\
Length/pressure & $S_{f}$ \\
Time & $S_{f}^{0.5}$ \\
\hline
\end{tabular}

$$
\begin{gathered}
\frac{U_{M}}{\sqrt{\sigma_{M} /\left(\rho_{M} L_{M}\right)}}=\frac{U_{F}}{\sqrt{\sigma_{F} /\left(\rho_{F} L_{F}\right)}}=\mathrm{Wn}, \\
\frac{U_{M} L_{M}}{\nu_{M}}=\frac{U_{F} L_{F}}{v_{F}}=\mathrm{Re},
\end{gathered}
$$

where $\sigma$ and $\nu$ are the surface tension, respectively. Thus, surface tension force also could be scaled in a scale model, although a surface tension of water $(0.07199 \mathrm{~N} / \mathrm{m})$ would negligibly low. In this study, viscosities of $8.899 \times 10^{-4} \mathrm{~kg} /$ $(\mathrm{m} \cdot \mathrm{s})$ and $1.831 \times 10^{-5} \mathrm{~kg} /(\mathrm{m} \cdot \mathrm{s})$ were used for water and air, respectively. Because the effects of the viscous forces are negligible, the same sloshing behavior could be predicted for the two models.

Previously published studies have shown that the frequency of excitation and amplitude in a pool affects the degree of nonlinearity in sloshing behavior; an increase in excitation results in violent responses from the fluid in the pool including turbulence, hydraulic jump, and wave breaking $[17,27]$. In this study, when a pool was excited with high-frequency excitation, such as $10 \mathrm{~Hz}$ for $1 / 8$ model and $7.071 \mathrm{~Hz}$ for $1 / 4$ model, differences in sloshing height was observed in the two models, while the exact same sloshing height was seen where the $1 / 8$ model was excited with $1 \mathrm{~Hz}$ and BDE. The difference is caused by hydraulic jump as shown in Figure 8. Thus, excessive excitation could result in a difference in the detail of the 


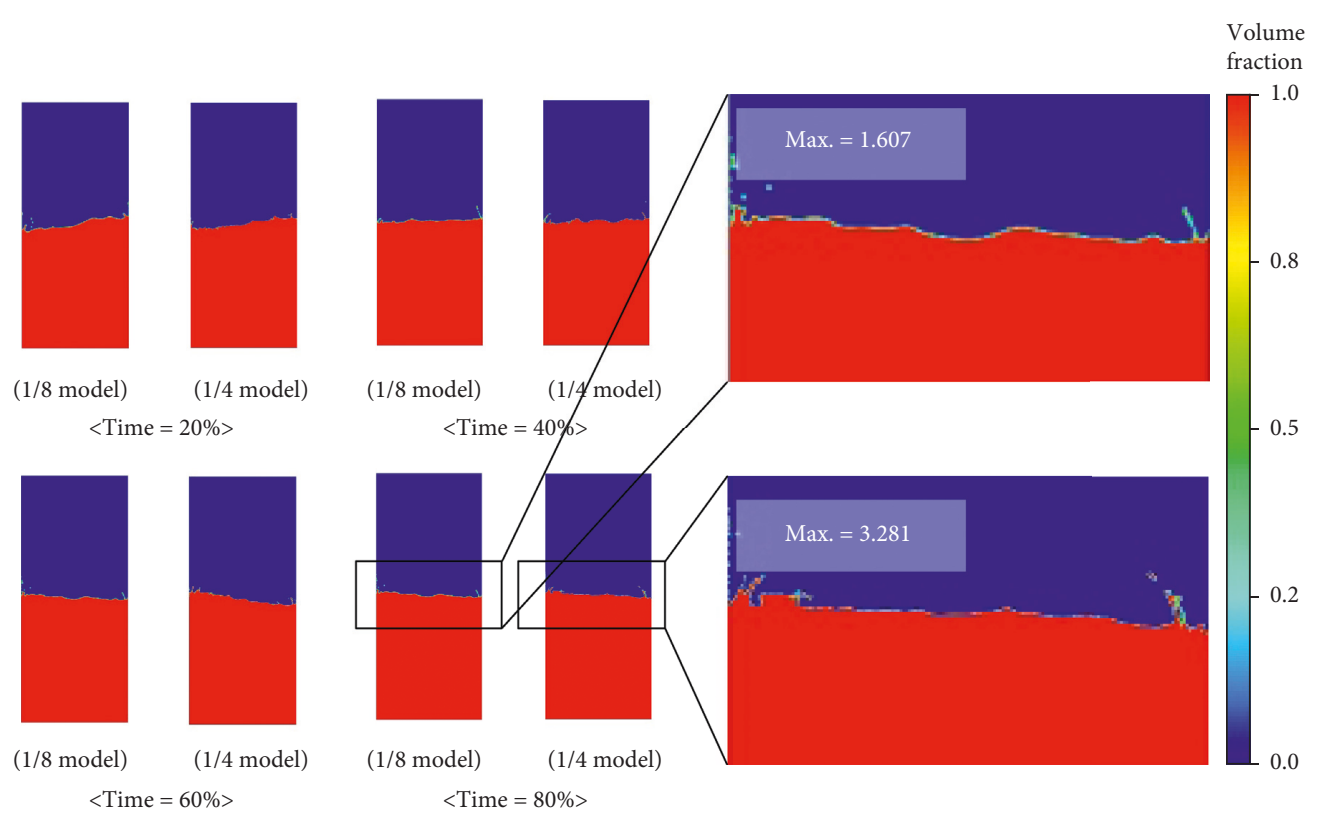

Figure 8: Differences in sloshing height caused by splash.

sloshing motion of the fluid. However, hydraulic jump does not affect the amount of fluid overflow or changes in the pressure on the wall; thus, the difference caused by nonlinearity in the behavior of the fluid seems to be negligible. The determined test conditions, in this study, for the scale models are therefore thought to be reasonable, though it is necessary to perform experimental studies to verify the results of this study.

There are several limitations in this study. An excessive increase in the height of the wall was used in the CFD model to prevent overflow from the pool, and overflow was not considered. Because the coolant in a pool removes the heat from the decay of the spent fuel assemblies and is used as a radiation shield [28], an investigation of the overflow of coolant is essential in a seismic assessment of a spent fuel pool $[2,29]$. Although overflow has not been considered in this study, it correlates with sloshing height. If the height could be predicted using a scale model, the overflow could also be predicted. Therefore, the scaling law could be adopted for the test while also taking into consideration the overflow of the liquid from a pool. The excitation runs along a horizontal direction in the two-dimensional CFD model. Because the calculated scaling law is coincident with the Froude scaling law, the same results were expected in the case of plane excitation using a three-dimensional model. Although an increase in the height of the surface of the free liquid affects the sloshing at the corner of a pool, it will not affect changes in the overall motion of the sloshing and pressure.

The calculated scaling law based on the natural frequency of the water in the pool is coincident with that of Froude similarity. The scaling law was validated via computational fluid dynamics analysis for the two models of different size. The results of this study showed that, even though excessive excitation may result in a partial difference in the sloshing behavior of the water with hydraulic jump and wave breaking, the scaling law could be adopted to predict the overall sloshing behavior, including free surface motion and pressure. Therefore, the authors believe that this study provides fundamental information for the seismic assessment of spent fuel pools using a scale model.

\section{Data Availability}

The data used to support the findings of this study are included within the article.

\section{Conflicts of Interest}

The authors declare that there are no potential conflicts of interest.

\section{Acknowledgments}

This work was supported by the Korea Institute of Energy Technology Evaluation and Planning (KETEP) and the Ministry of Trade, Industry and Energy (MOTIE) of the Republic of Korea (No. 20171510101920).

\section{References}

[1] U.S.N.R. Commission, Consequence Study of a Beyond-Design-Basis Earthquake Affecting the Spent Fuel Pool for a U.S. Mark I Boiling Water Reactor, NUREG-2161, Washington, DC, USA, 2014.

[2] M. Baba, "Fukushima accident: what happened?," Radiation Measurements, vol. 55, pp. 17-21, 2013.

[3] K. Shozugawa, N. Nogawa, and M. Matsuo, "Deposition of fission and activation products after the Fukushima Dai-ichi nuclear power plant accident," Environmental Pollution, vol. 163, pp. 243-247, 2012.

[4] Y. Zhao, P. R. Wilson, and J. D. Stevenson, "Nonlinear 3-D dynamic time history analysis in the reracking modifications for a nuclear power plant," Nuclear Engineering and Design, vol. 165, no. 1-2, pp. 199-211, 1996. 
[5] O. M. Faltinsen, "A numerical nonlinear method of sloshing in tanks with two-dimensional flow," JSH Statement, vol. 22, pp. 193-202, 1978.

[6] J. B. Frandsen, "Sloshing motions in excited tanks," Journal of Computational Physics, vol. 196, no. 1, pp. 53-87, 2004.

[7] R. L. Frano, "Evaluation of the fluid-structure interaction effects in a lead-cooled fast reactor," Nuclear Technology, vol. 189, no. 1, pp. 1-10, 2015.

[8] F. Axisa and J. Antunes, Modelling of Mechanical Systems, Fluid-Structure Interaction, Boston, MA, USA, 2007.

[9] G. X. Wu, Q. W. Ma, and R. E. Taylor, "Numerical simulation of sloshing waves in a $3 \mathrm{D}$ tank based on a finite element method," Applied Ocean Research, vol. 20, no. 6, pp. 337-355, 1998.

[10] W. Chen, M. A. Haroun, and F. Liu, "Large amplitude liquid sloshing in seismically excited tanks," Earthquake Engineering \& Structural Dynamics, vol. 25, no. 7, pp. 653-669, 1996.

[11] T. Okamoto and M. Kawahara, "Two-dimensional sloshing analysis by Lagrangian finite element method," International Journal for Numerical Methods in Fluids, vol. 11, no. 5, pp. 453-477, 1990.

[12] M. A. Goudarzi and S. R. Sabbagh-Yazdi, "Investigation of nonlinear sloshing effects in seismically excited tanks," Soil Dynamics and Earthquake Engineering, vol. 43, pp. 355-365, 2012.

[13] S. Mitra and K. P. Sinhamahapatra, "Slosh dynamics of liquidfilled containers with submerged components using pressurebased finite element method," Journal of Sound and Vibration, vol. 304, no. 1-2, pp. 361-381, 2007.

[14] S.-Y. Kim, Y. Kim, and J. Lee, "Comparison of sloshing-induced pressure in different scale tanks," Ships and Offshore Structures, vol. 12, no. 2, pp. 244-261, 2017.

[15] A. G. Merino, L. C. de la Peña, and A. González, "Experimental validation of the seismic analysis methodology for free-standing spent fuel racks," Nuclear Engineering and Technology, vol. 51, no. 3, pp. 884-893, 2019.

[16] T. Futatsugi, C. Yanagi, M. Murase, S. Hosokawa, and A. Tomiyama, "Countercurrent air-water flow in a scale-down model of a pressurizer surge line," Science and Technology of Nuclear Installations, vol. 2012, Article ID 174838, 7 pages, 2012.

[17] H. Akyildiz and N. E. Ünal, "Sloshing in a three-dimensional rectangular tank: numerical simulation and experimental validation," Ocean Engineering, vol. 33, pp. 2135-2149, 2006.

[18] J. H. Jung, H. S. Yoon, and C. Y. Lee, "Effect of natural frequency modes on sloshing phenomenon in a rectangular tank," International Journal of Naval Architecture and Ocean Engineering, vol. 7, no. 3, pp. 580-594, 2015.

[19] M. Ha, D. Kim, H. I. Choi, C. Cheong, and S. H. Kwon, "Numerical and experimental investigations into lliquid sloshing in a rectangular tank," in Proceedings of the 2012 World Congress on Advances in Civil, Environmental, and Materials Research (ACEM'12), Seoul, Republic of Korea, August 2012.

[20] B. Godderidge, S. Turnock, M. Tan, and C. Earl, "An Investigation of Multiphase CFD Modelling of a Lateral Sloshing Tank," Computers \& Fluids, vol. 38, 2009.

[21] S. Siddapureddy and A. K. Satapathy, "Liquid sloshing in a moving tank-CFD," in Proceedings of the 6th International o 43rd National Conference on Fluid Mechanics and Fluid Power, Allahabad, Uttar Pradesh, December 2016.

[22] R. 2 Regulatory Guide No. 1.60, Design Response Spectra for Seismic Design of Nuclear Power Plants, R. 2 Regulatory Guide No. 1.60, Washington, DC, USA, 2014.
[23] V. Heller, "Scale effects in physical hydraulic engineering models," Journal of Hydraulic Research, vol. 49, no. 3, pp. 293-306, 2011.

[24] D. Lu, X. Zeng, J. Dang, and Y. Liu, “A calculation method for the sloshing impact pressure imposed on the roof of a passive water storage tank of AP1000," Science and Technology of Nuclear Installations, vol. 2016, p. 8, 2016.

[25] W. Sheng, R. Alcorn, and T. Lewis, "Physical modelling of wave energy converters," Ocean Engineering, vol. 84, pp. 29-36, 2014.

[26] S. A. Hughes, "Physical models and laboratory techniques in coastal engineering," in Advanced Series on Ocean Engineering, World Scientific, Singapore, 1994.

[27] M. Eswaran and U. K. Saha, "Sloshing of liquids in partially filled tanks-a review of experimental investigations," Ocean Systems Engineering, vol. 1, no. 2, pp. 131-155, 2011.

[28] H. Wang, L. Ge, J. Shan, J. Gou, and B. Zhang, "Safety analysis of CPR1000 spent fuel pool in case of loss of heat sink," in Proceedings of the International Conference on Nuclear Engineering (ICONE), Chengdu, China, January 2013.

[29] T.-M. Shin, "Safety review of severe accident senario for wet spent fuel storage facility," Journal of the Korean Radioactive Waste Society, vol. 9, no. 4, pp. 231-236, 2011. 

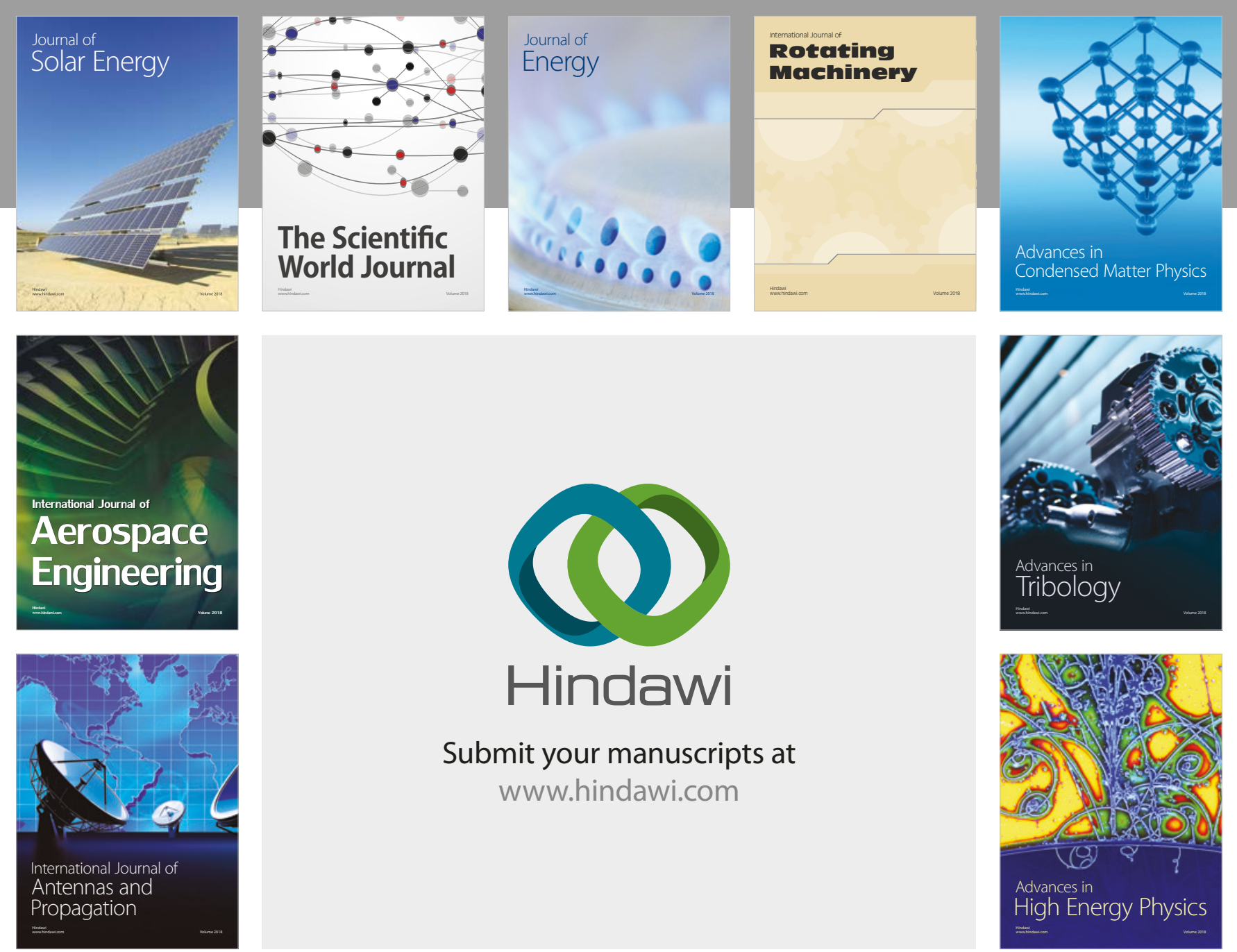

Submit your manuscripts at

www.hindawi.com
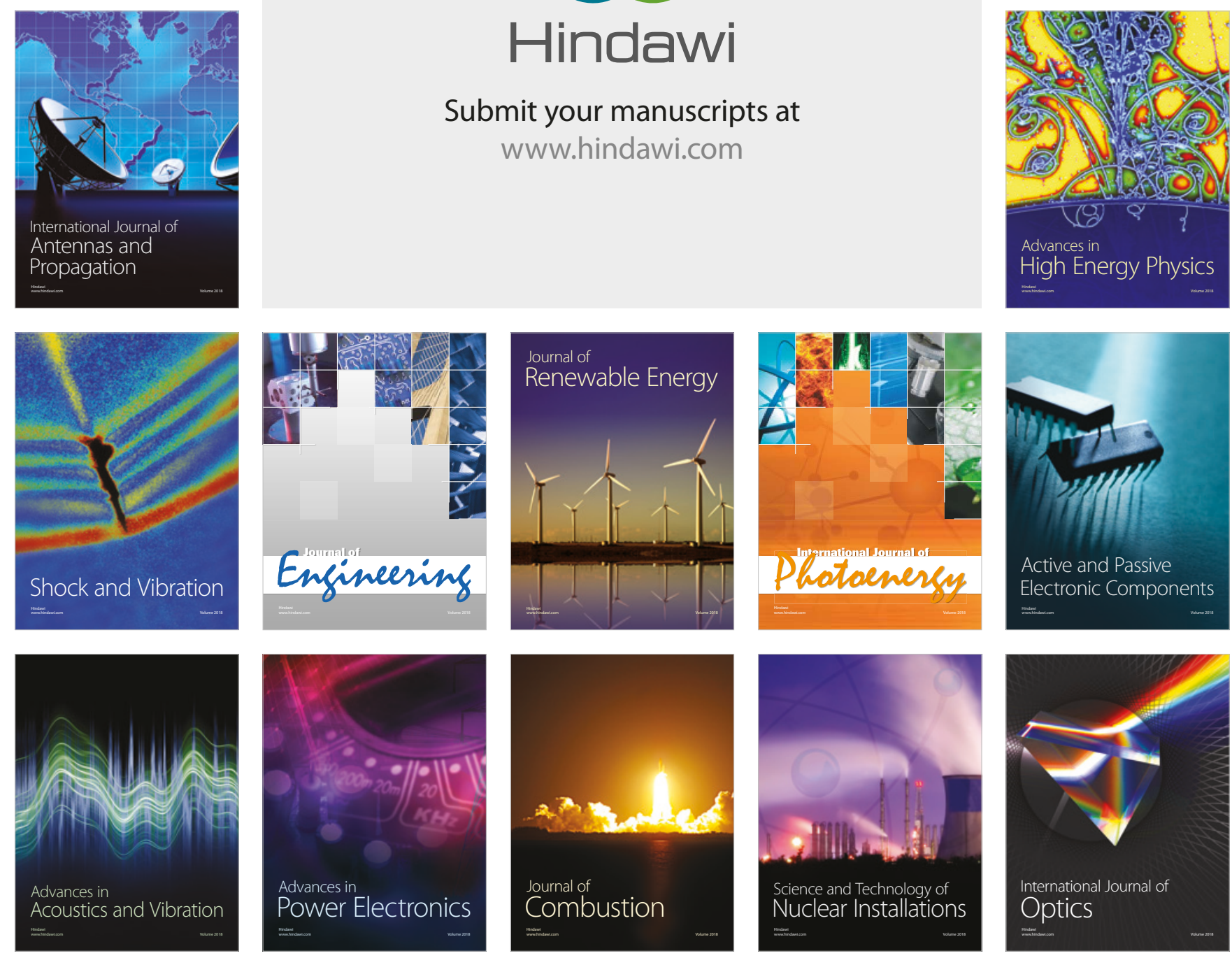